\title{
Oral Microbiota Development in Early Childhood
}

\author{
Beatrice Kennedy $\mathbb{1}^{1 *}$, Sari Peura ${ }^{1,2}$, Ulf Hammar ${ }^{1}$, Silvia Vicenzi ${ }^{1,3}$, Anna Hedman ${ }^{4}$, \\ Catarina Almqvist ${ }^{4,5}$, Ellika Andolf ${ }^{6}$, Göran Pershagen ${ }^{7,11}$, Johan Dicksved ${ }^{8}$, \\ Stefan Bertilsson ${ }^{9,10}$ \& Tove Fall (i) ${ }^{1}$
}

Early life determinants of the oral microbiota have not been thoroughly elucidated. We studied the association of birth and early childhood characteristics with oral microbiota composition using $16 \mathrm{~S}$ ribosomal RNA ( $r R N A)$ gene sequencing in a population-based Swedish cohort of 59 children sampled at 6,12 and 24 months of age. Repeated-measurement regression models adjusted for potential confounders confirmed and expanded previous knowledge about the profound shift of oral microbiota composition in early life. These alterations included increased alpha diversity, decreased beta diversity and alteration of bacterial composition with changes in relative abundance of 14 of the 20 most common operational taxonomic units (OTUs). We also found that birth characteristics, breastfeeding and antibiotic use were associated with overall phyla distribution and/or with the relative abundance of specific OTUs. Further, we detected a novel link between morning salivary cortisol level, a physiological marker of neuroendocrine activity and stress, and overall phyla distribution as well as with decreased abundance of the most common OTU mapped to the Streptococcaceae family. In conclusion, a major part of the maturation of the oral microbiome occurs during the first two years of life, and this development may be influenced by early life circumstances.

The oral microbiota represents a complex, dynamic, and heterogeneous bacterial ecosystem ${ }^{1}$, and its composition has been discussed in relation to oral health ${ }^{2,3}$ and to cardiovascular disease ${ }^{4}$. However, recent studies using $16 \mathrm{~S}$ ribosomal RNA (rRNA) sequencing have also linked the oral microbiome in childhood to allergy and asthma diagnoses ${ }^{5}$, weight gain trajectory ${ }^{6}$, and autism spectrum disorder ${ }^{7}$.

The 16S rRNA amplicon sequencing approach has also been employed to study the neonatal oral microbiota development trajectory, and associations have been noted with antibiotic treatment during pregnancy ${ }^{8}$, gestational age ${ }^{9,10}$, mode of delivery ${ }^{11-13}$ and neonatal $\operatorname{diet}^{14}$. Few longitudinal $16 \mathrm{~S}$ rRNA sequencing studies have however investigated the oral microbiota development during subsequent early childhood ${ }^{15,16}$. A recent Swedish longitudinal study reported an association between mode of delivery and oral microbial community composition at 3 and 6 months of age ${ }^{17}$, as well as more long-standing associations between partial breastfeeding and early life antibiotic treatment with oral microbiota composition until 7 years of age. However, the participants in that study all had a family history of allergic disease, and a study of potential determinants of oral microbiota development in early childhood in a population-based setting is warranted.

Further, measures of salivary cortisol mirror the biologically active cortisol level in the body, and have been shown to reflect individual stress reactivity and level of acute and/or chronic stress ${ }^{18}$. In smaller cross-sectional studies, salivary cortisol has been associated with the presence of cariogenic bacteria in the oral cavity, as assessed through bacterial cultures, and with the development of dental caries in children ${ }^{19-21}$. No previous study has however investigated the association between salivary cortisol and oral microbiota community development.

\footnotetext{
${ }^{1}$ Department of Medical Sciences, Molecular Epidemiology and Science for Life Laboratory, Uppsala University, Uppsala, Sweden. ${ }^{2}$ Department of Forest Mycology and Plant Pathology, Science for Life Laboratory, Swedish University of Agricultural Sciences, Uppsala, Sweden. ${ }^{3}$ School of Medicine, University of Tasmania, Hobart, Australia. ${ }^{4}$ Department of Medical Epidemiology and Biostatistics, Karolinska Institutet, Stockholm, Sweden. ${ }^{5}$ Unit of Pediatric Allergy and Pulmonology at Astrid Lindgren Children's Hospital, Karolinska University Hospital, Stockholm, Sweden. ${ }^{6}$ Department of Clinical Sciences, Danderyd Hospital, Stockholm, Sweden. ${ }^{7}$ Institute of Environmental Medicine, Karolinska Institutet, Stockholm, Sweden. ${ }^{8}$ Department of Animal Nutrition and Management, Swedish University of Agricultural Sciences, Uppsala, Sweden. ${ }^{9}$ Department of Ecology and Genetics, Limnology, Uppsala University, Uppsala, Sweden. ${ }^{10}$ Department of Aquatic Sciences and Assessment, Swedish University of Agricultural Sciences, Uppsala, Sweden. ${ }^{11}$ Centre for Occupational and Environmental Medicine, Region Stockholm, Stockholm, Sweden. *email: beatrice.kennedy@medsci.uu.se
} 
In this longitudinal population-based cohort study, we combined results from repeated saliva sampling with prospectively collected information from questionnaires and from national birth and prescription registers. The overall scope of the study was to explore early life determinants for the oral microbiota development trajectory including maternal microbiota, birth characteristics, breastfeeding, antibiotic treatment and exposure to a furry pet. In addition, we obtained the results from repeated analyses of morning salivary cortisol, which uniquely allowed us to investigate the potential association between salivary cortisol and oral microbiota in young children.

\section{Material and Methods}

The Born into Life cohort. The Born into Life cohort is a prospective longitudinal birth cohort led and maintained by researchers at Karolinska Institutet in Sweden ${ }^{22}$. The main initial scope of the Born into Life cohort was to investigate how maternal, neonatal, and early life circumstances may influence somatic health in childhood and beyond. To this end, women previously enrolled in the LifeGene study ${ }^{23}$, who were living in the Stockholm County Council area and who became pregnant between 2010 and 2012 were invited to participate. Time-points for questionnaires and biomarker sampling were pre-determined before the initiation of the Born into Life cohort. The time-point at gestational week 26-28 was chosen in order to assess women late in pregnancy, but not too close to full term as this would entail a risk of missing women giving pre-term birth. The time-points for the child, at ages 6, 12 and 24 months, were arbitrary time-points chosen to be spread evenly across the first two years of life. There were investigation at other time-points, but salivary samples were not included at those assessments. The maternal cohort comprised of 107 pregnant women, and 93 children were included in the overall Born into Life child cohort at birth. We excluded 28 mothers and 27 children that did not participate in any follow-up after birth.

Questionnaire data. Mothers responded to questionnaires at inclusion in LifeGene as well as during pregnancy at gestational week 26-28. The mother or father responded to Born into Life questionnaires at child age 6,12 and 24 months. We extracted information on maternal highest attained education level from the LifeGene questionnaire. We obtained information on number of months of exclusive breastfeeding (categorized as $0-2,3-4$, or $5+$ ) from the Born into Life questionnaire at 6 months. Subsequent information on exclusive breastfeeding from the later questionnaires at 12 and 24 months were not included as the upper age-range of the pre-determined response categories remained $5+$ months even at subsequent follow-up. We also included time-updated information on exposure to furry pet, defined as presence of a cat or a dog in the household, from the Born info Life questionnaires at 6, 12 and 24 months.

National health register data. All residents in Sweden have a unique personal identification number, given at birth or immigration, which enabled record linkages of the mothers and their children to Swedish national health registers. From the Medical Birth Register we obtained information on maternal body mass index (BMI) and maternal smoking in the first trimester, parity (nullipara or multipara), and birth characteristics including the infant's sex, day of birth, mode of delivery (vaginal or caesarean), gestational age in weeks, and birthweight in grams. Time-updated information on prescription of antibiotics to the child was extracted from the Swedish Prescribed Drug Register. Use of antibiotics was analysed both as time-updated ever-use, and use within the last 90 days at saliva sampling occasion.

Saliva samples. Evening and morning saliva samples were collected from the children at 6, 12, and 24 months of age, and from the mothers at gestational week 26-28 (for sample instructions see Supplementary Information). Once the samples had been handed in, they were centrifuged for two minutes, transported at standard refrigerator temperature and then stored at $-20^{\circ} \mathrm{C}$ until cortisol analysis ${ }^{22}$. After cortisol analysis, the remaining sample was stored at $-80 \mathrm{C}$.

Cortisol assessment. Salivary cortisol concentration were analysed at the Centre for Child Research, Stockholm South General Hospital, Stockholm, Sweden, using the standardised CORT-CT2 radioimmunoassay kit (Cisbio Bioassays, Codolet, France) according to the manufacturer's instructions. The detection interval ranged from concentrations of $1-100 \mathrm{nmol} / \mathrm{L}$. Inter-assay and intra-assay variations were below $5 \%$. Each sample was analysed twice and the mean value of these were used for analysis ${ }^{22}$. Salivary cortisol level was log-transformed in all analyses. As the microbiota analysis was performed in evening saliva samples, we only included morning salivary cortisol as anexposure in our regression analyses, as we wanted to reduce the risk of potential bias from a matrix effect. The saliva matrix refers to the overall saliva composition and all other components of a saliva sample than the analytes of interest, i.e water concentration and $\mathrm{pH}$ level, or presence of food or drug residues ${ }^{24}$. Analysing both cortisol and microbiota from the same saliva samples may thereby introduce spurious associations due to sample confounding.

Microbiota analysis and quality control. Prior to DNA extraction, the analysis order was randomized across samples. Subsequently, DNA from 241 evening saliva samples (156 samples from children, 85 samples from mothers) and 5 negative controls was extracted using MoBio PowerSoil DNA kit (Mobio, Solana beach, CA, USA). After sequencing of the v3 and v4 regions of $16 \mathrm{~S}$ rRNA regions (for PCR protocol see Supplementary Information), the samples were purified with AMPure, quantified using Qubit (ThermoFisher Scientific) with dsDNA high-sensitivity assay. 216 barcoded samples with individual dual indices passed this QC step and were pooled in equimolar amounts into two pools of 106 and 110 samples each. The amplicons were sequenced using the Illumina MiSeq technology $(2 * 300$ cycles) at Science for Life Laboratory (Uppsala University, Sweden). The resulting reads were processed using Mothur ${ }^{25}$, following the standard operation protocol ${ }^{26}$, except for operational taxonomic unit (OTU) clustering, which was done using Vsearch (abundance-based greedy clustering; ${ }^{27}$ ) as implemented in Mothur. All samples with less than 10472 sequences were removed $(n=1)$ and the remaining samples were rarefied to this sequencing depth using Mothur. A cut-off of $97 \%$ identity between sequences was used to delineate OTUs. The classification of the operational taxonomic units (OTUs) was done using the Silva database (version 


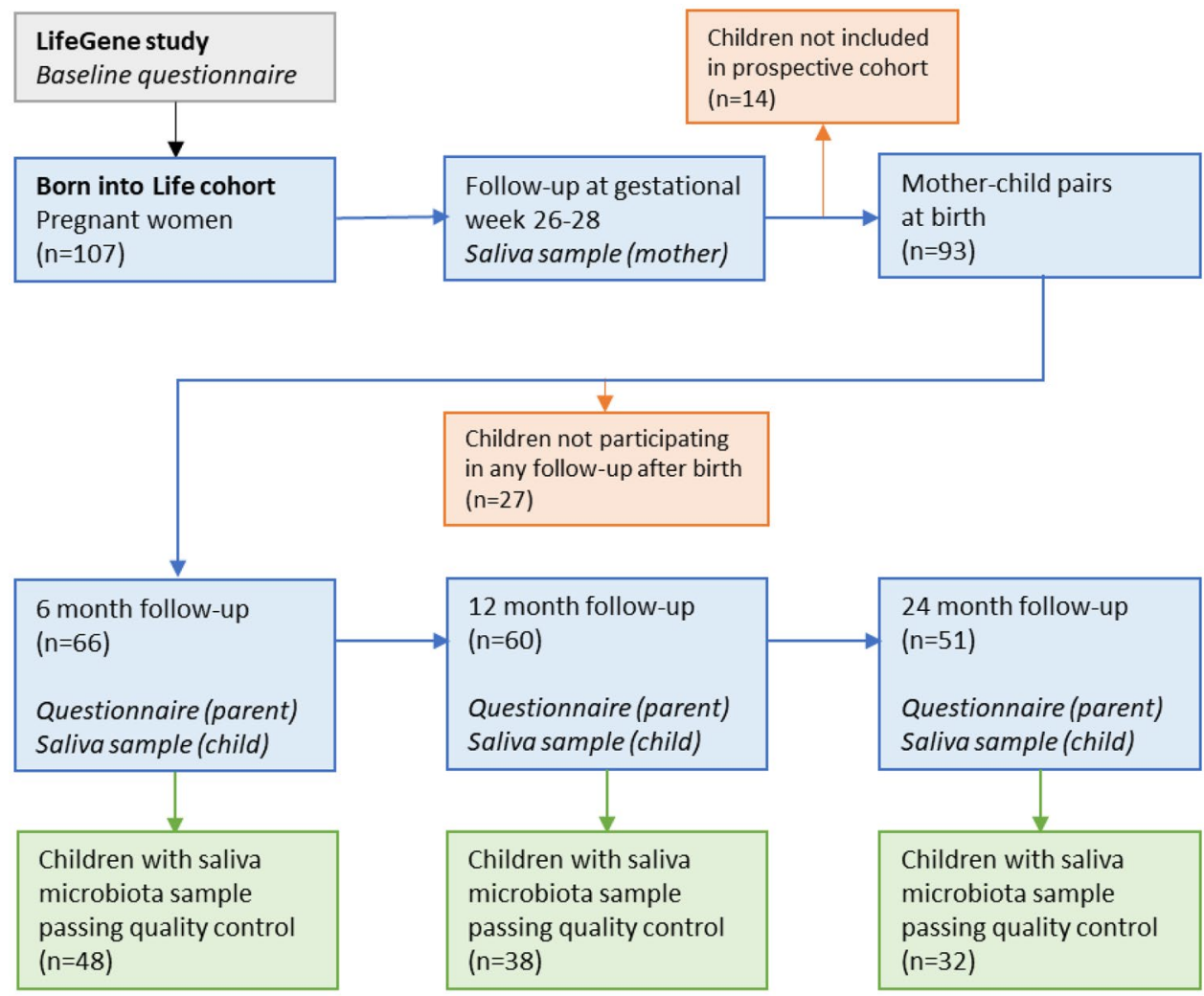

Study population $(n=59)$

Figure 1. Flowchart of the study population.

128). In total we detected 24539 OTUs, whereof 5224 in more than one sample. Sequences included in our analyses has been submitted to the Sequence Read Archive (SRA) open repository under reference number PRJNA575550.

Study population. Fourteen saliva samples from children had an abnormally low diversity and community composition, possibly implying contamination, and those were accordingly removed from the dataset. After all exclusions, 118 samples from 59 children remained, as well as 55 samples from their mothers. These 59 children constituted our final study population (Fig. 1).

Statistical analysis. Strategy. We applied a series of repeated-measurement regression models to evaluate the association of each of a set of exposures on four different measures of microbiota composition. Since the separate exposure models might require adjustments for different sets of covariates, we created a directed acyclic graph (DAG) ${ }^{28}$ prior to the analysis phase. The DAG is a graphical presentation of the theoretical framework and potential underlying causal structures connecting exposures and covariates with the microbiota outcome in our study (Fig. S1). The hypothesized causal structure is based on previous literature. Using the DAG, we could apply the d-separation criteria and identify the potential confounders for each model (Table 1), as suggested by Greenland et al. ${ }^{29}$. The results from all models were then evaluated jointly for statistical significance using a false discovery rate (FDR) of $10 \%$, where FDR was calculated using the Benjamini-Hochberg method ${ }^{30}$. All data was de-identified centrally before data delivery to the researchers and all statistical analyses were performed using Stata v.14 (Stata, College Station, TX, USA).

Models and outcomes. In the main analyses, continuous exposures were modelled using restricted cubic splines with three knots, to account for possible non-linearity in the relationship with the outcomes. Knots were placed a priori according to Harrell's recommendations ${ }^{31}$.

We considered four different aspects of oral microbiota composition. The association of all exposures with (1) alpha diversity, assessed by the inverse Simpson index ${ }^{32}$, was analysed using linear mixed models with restricted maximum likelihood and participant ID as random effect. For (2) overall composition based on beta diversity, assessed through non-metric multidimensional scaling (NMDS) calculation ${ }^{33-36}$ using Bray-Curtis distances with the R package vegan based on the rarefied data, we used bivariate linear mixed effects model with participant-ID as a random effect, evaluating both NMDS-axes jointly. This model was fitted by using the generalized structural equation command gsem in Stata. The test for association between mother's beta diversity and child's beta diversity at 6, 12 and 24 months was performed using multivariate regression, jointly evaluating both NMDS-axes. The outcome (3) proportion of each bacterial phylum was analysed using fractional multinomial logistic models 


\begin{tabular}{|l|l|}
\hline Exposure & Confounders \\
\hline Age of child & - \\
\hline Maternal oral microbiota & Age, maternal age, maternal BMI* \\
\hline Parity & Age, maternal age. \\
\hline Gestational age & Age, maternal education level* \\
\hline Birth weight & Age, maternal BMI, maternal education level, parity, sex, gestational age* \\
\hline Mode of delivery & Age, maternal BMI, parity, gestational age, birth weight \\
\hline Exclusive breastfeeding & $\begin{array}{l}\text { Age, maternal age, maternal education level, parity, mode of delivery, } \\
\text { gestational age, birth weight* }\end{array}$ \\
\hline Morning salivary cortisol level & Age, maternal education level, gestational age, birth weight* \\
\hline Antibiotic treatment (within 90 days or ever) & Age, maternal education level, sex, gestational age \\
\hline Exposure to furry pet & Age, maternal age, maternal education level \\
\hline
\end{tabular}

Table 1. Covariates included in the different models. *We identified maternal smoking during pregnancy as a potential confounder. However, in our maternal cohort no women reported smoking whilst pregnant, and this variable is therefore not included in the model. BMI: Body Mass Index.

with clustered robust standard errors ${ }^{37}\left(\right.$ fmlogit $\left.^{38}\right)$ with participant identity as cluster, which is a way of modelling proportional outcomes. The proportions of each bacterial phyla in the saliva were calculated for phyla present in at least $75 \%$ of the samples, and all phyla were evaluated jointly. For the (4) relative abundance of the 20 most common OTUs (Table S1), the 20 most common OTUs were selected by rank-transforming OTU abundance within each sample (with absent OTUs given the lowest possible rank), and choosing the 20 OTUs with the highest average rank across all samples. The relative abundance of each OTU was analysed using fractional logistic regression, again with participant identity as cluster. The latter model yielded relative proportion ratios (RPR). An RPR $>1$ indicates a higher relative abundance of the OTU in exposed group compared to non-exposed, and $\mathrm{RPR}<1$ a lower relative abundance.

To specifically evaluate the maternal salivary microbiota composition as an exposure, the maternal and child alpha diversity, beta diversity, proportions of phyla, and 20 most common OTUs were compared, respectively (e.g. maternal alpha diversity was explored in association with child alpha diversity).

Sensitivity analyses. We used ordered logistic regression (or, for multivariate outcomes, ordinal generalized structural equations) with cluster-robust standard errors for all exposure-outcome associations as a sensitivity analysis. Ordered logistic regression is a semi-parametric method which generalizes the Mann-Whitney test ${ }^{31}$. Any findings where FDR was below $10 \%$ in the main analysis but the sensitivity analysis had a p-value $>0.05$ were assumed to be false positive and were not considered or discussed further.

In addition, we also analysed beta diversity using the permutational multivariate ANOVA (PERMANOVA) method $^{39}$, as implemented in the R-package vegan. We used the wrapper function adonis_II from the R-package RVaideMemoire ${ }^{40}$ to get type II sum of squares. Independence was assumed, due to individuals having non-equal number of observations. We determined significance using the FDR cut-off from the main analyses, corresponding to a p-value of 0.0209. These results are shown in the Supplementary material (Table S4).

Post-hoc tests. For alpha diversity as well as for the 20 most common OTU:s we added an interaction term between exposure and age to all models with a exposure-outcome association passing the significance thresholds. If the p-value for this interaction term was below 0.05 , interaction was deemed present. If an exposure was associated with distribution of bacterial phyla proportions, we carried out post-hoc tests, testing all phyla present in more than $75 \%$ of the samples individually using fractional logistic regression with cluster-robust standard errors. These post-hoc tests were not corrected for multiple comparisons. Associations with a p-value $<0.05$ in both main and sensitivity analysis were deemed significant.

Cladogram. We used the cladogram functionality of the Linear discriminant analysis Effect Size (LEfSe) method ${ }^{41}$ as implemented in Galaxy ${ }^{42}$ to illustrate differences in saliva microbiota composition between 6 and 24 months. In the cladogram, differences between the two time-points are illustrated at phyla, class, order, family, and genus levels.

Ethical approval. This study was approved by the Regional Ethical Review Board in Stockholm, Sweden (DNR 2011/192-31/2, with addendums), and all research was performed in accordance with all relevant guidelines and regulations. All women in the Born into Life cohort gave informed consent at enrolment during pregnancy, and both parents gave additional informed consent for the child.

\section{Results}

The final study population comprised of 59 children. Maternal median age at birth was 31.9 years (Table 2). The majority of mothers had a university level education, were nulliparous, and had a normal BMI during the first trimester. None reported smoking during pregnancy. Of the 59 children, 22 were girls and 37 were boys. Overall, 12 children were delivered with a caesarean section. Three children were born preterm ( $<37$ weeks) and two post-term ( $\geq 42$ weeks). The median birth weight was 3560 grams. 


\begin{tabular}{|c|c|}
\hline \multicolumn{2}{|l|}{ Maternal characteristics } \\
\hline Age at childbirth, years (IQR) & $31.9(29.7-34.3)$ \\
\hline \multicolumn{2}{|l|}{ University level education (\%) } \\
\hline Yes & $51(86)$ \\
\hline No & $8(14)$ \\
\hline \multicolumn{2}{|l|}{$\mathrm{BMI}$ in the first trimester, $\mathrm{kg} / \mathrm{m}^{2}(\%)$} \\
\hline Underweight $(<18.5)$ & $2(3 \%)$ \\
\hline Normal weight (18.5-24.9) & $41(69 \%)$ \\
\hline Overweight (25.0-29.9) & $13(22 \%)$ \\
\hline Obese $(\geq 30.0)$ & $3(5 \%)$ \\
\hline Smoking during pregnancy (\%) & $0(0)$ \\
\hline \multicolumn{2}{|l|}{ Parity (\%) } \\
\hline Nullipara & $41(69)$ \\
\hline Multipara & $18(31)$ \\
\hline \multicolumn{2}{|l|}{ Birth characteristics } \\
\hline \multicolumn{2}{|l|}{$\operatorname{Sex}(\%)$} \\
\hline Female & $22(37)$ \\
\hline Male & $37(63)$ \\
\hline \multicolumn{2}{|l|}{ Mode of delivery (\%) } \\
\hline Vaginal & $47(80)$ \\
\hline Caesarean & $12(20)$ \\
\hline Birth weight, grams (IQR) & $3560(3240-3825)$ \\
\hline \multicolumn{2}{|l|}{ Gestational age (\%) } \\
\hline Preterm ( $<37$ weeks) & $3(5 \%)$ \\
\hline Full term (37-41 weeks) & $54(92 \%)$ \\
\hline Post term ( $\geq 42$ weeks) & $2(3 \%)$ \\
\hline
\end{tabular}

Table 2. Baseline characteristics of the 59 mother-child pairs included in the study IQR: Interquartile range. BMI: Body Mass Index.

\begin{tabular}{|c|c|c|c|}
\hline & 6 months & 12 months & 24 months \\
\hline Number of children & 48 & 38 & 32 \\
\hline Age, months (IQR) & $6.6(6.1-7.0)$ & $12.3(11.8-12.8)$ & $24.5(23.9-24.7)$ \\
\hline Antibiotic treatment within 90 days (\%) & $3(6)$ & $1(3)$ & $5(16)$ \\
\hline Antibiotic treatment, ever-use (\%) & $3(6)$ & $2(5)$ & $9(28)$ \\
\hline Morning salivary cortisol, nmol/L (IQR) & $24.0(19.0-34.0)^{\mathrm{a}}$ & $28.5(17.4-37.4)^{\mathrm{b}}$ & $23.3(14.4-30.8)^{\mathrm{c}}$ \\
\hline Furry pet present in household (\%) & $1(2)^{\mathrm{d}}$ & $3(19)^{\mathrm{e}}$ & $4(31)^{\mathrm{f}}$ \\
\hline \multicolumn{4}{|c|}{ Age when exclusive breastfeeding was discontinued (\%)* } \\
\hline $0-2$ months & $4(7)$ & & \\
\hline 3-4 months & $14(24)$ & & \\
\hline $5+$ months & $31(53)$ & & \\
\hline
\end{tabular}

Table 3. Study population characteristics at follow-up IQR: Interquartile range. ${ }^{a} n=42 .{ }^{b} n=34 .{ }^{c} n=28$. ${ }^{d} n=42 .{ }^{e} n=16 .{ }^{f} n=13 . *$ Column percentages do not add to total due to missing data.

At follow-up visits (Table 3), a dispensed antibiotic prescription within 90 days before the visit was noted for $6 \%, 3 \%$, and $16 \%$ of the children (at ages 6,12 and 24 months, respectively). The cumulative incidence of ever having been prescribed antibiotic treatment reached $28 \%$ at age 24 months, and the two types of antibiotics most commonly prescribed were the $\beta$-lactam antibiotics phenoxylmethylpenicillin and amoxicillin $(65 \%$ and $15 \%$ of all prescriptions, respectively). A furry pet was present in 2, 19 and $31 \%$ of the households responding to this question (at ages 6, 12 and 24 months, respectively).

Age of child. Age of the child was positively associated with alpha diversity, with a nearly threefold increase noted from the first saliva sampling at 6 months to the sampling at 24 months $(p<0.001$, Fig. 2). Increasing age was also associated with the overall distribution of phyla $(\mathrm{p}<0.001)$, with the alterations in abundance of the phyla Firmicutes, Proteobacteria, Epsilonbacteraoeta (phyl. nov.) and Fusobacteria specifically identified in post-hoc analyses and also confirmed in semi-parametric sensitivity analyses as associated with age (all p $<0.001$, Fig. 2 and Table S3). Overall, at 6 months, the phyla Firmicutes vastly dominated the oral microbiota, with a subsequent decrease noted both at 12 months and at 24 months (Fig. 3). The microbiota composition development between 6 and 24 months, across all investigated taxonomic levels, is depicted in the cladogram (Fig. 4). 

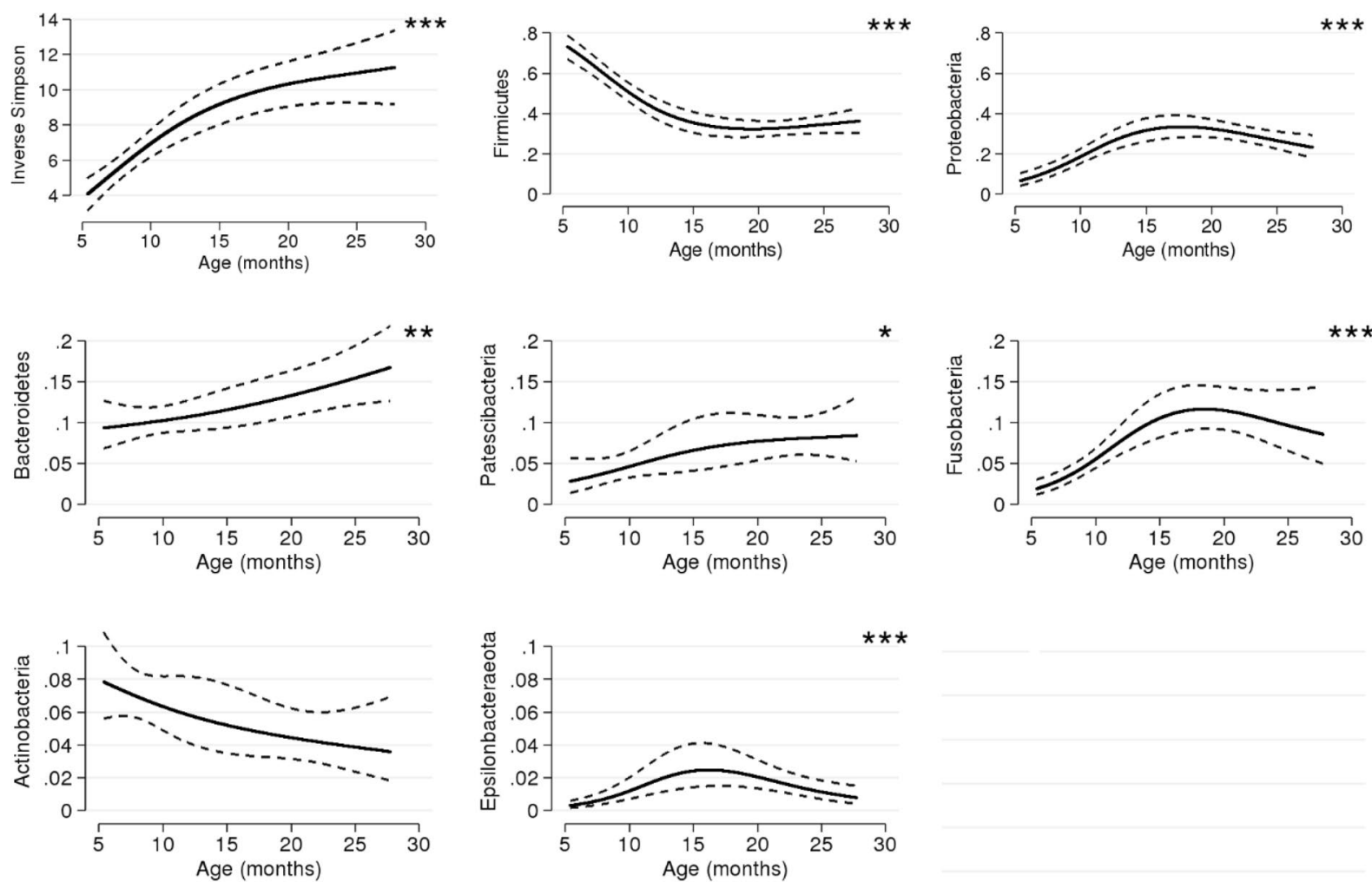

Figure 2. Model-based predictions of inverse Simpson and proportion of phyla plotted against age. Lines indicate mean values and $95 \%$ confidence intervals. The $y$-axis shows the relative abundance in proportion. $* \mathrm{p}<0.05, * * \mathrm{p}<0.01, * * * \mathrm{p}<0.001$.

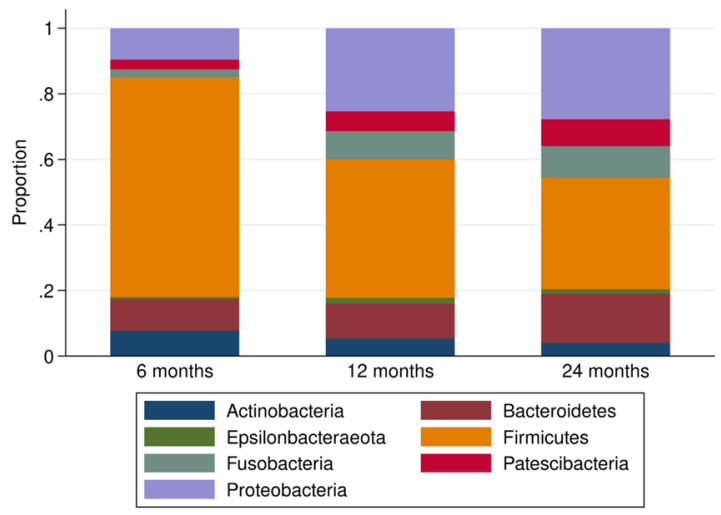

Figure 3. Stacked bar charts of phyla distribution at 6, 12, and 24 months, respectively.

At the more highly resolved taxonomic levels, age was associated with 14 of the 20 most common OTUs explored (Fig. 5 and Fig. S2). The decreasing Firmicutes abundance noted at 12 and 24 months was here represented by steep reductions in OTUs belonging to the Streptococcaceae family (OTU0001, OTU0009, OTU00019, Table S2) and decreases of OTUs in the Veillonella genus or the Veillonellaceae family (OTU0002, OTU0011, and OTU0020, respectively). However, one initially rare OTU mapped to the Streptococcaceae family (OTU00052) increased with increasing age. Within the Bacteroidetes phyla, Porphyromonas (OTU00012) also increased with age, whereas two common OTUs within the Proteobacteria phyla belonging to the Pasteurellaceae (OTU00003) and Neisseriaceae (OTU00004) families, respectively, both exhibited inverted u-shaped associations with age. We furthermore noted an association between age and an OTU mapped as Fusobacterium genus (OTU0007).

In addition, we also noted a decreased beta diversity $(\mathrm{p}<0.001$, Fig. 6$)$.

Maternal oral microbiota. The maternal oral microbiota composition during pregnancy was not associated with early childhood oral microbiota. However, when we compared the child cohort across all time points with the maternal cohort, we noted that that the children became more similar to an adult composition with increasing 


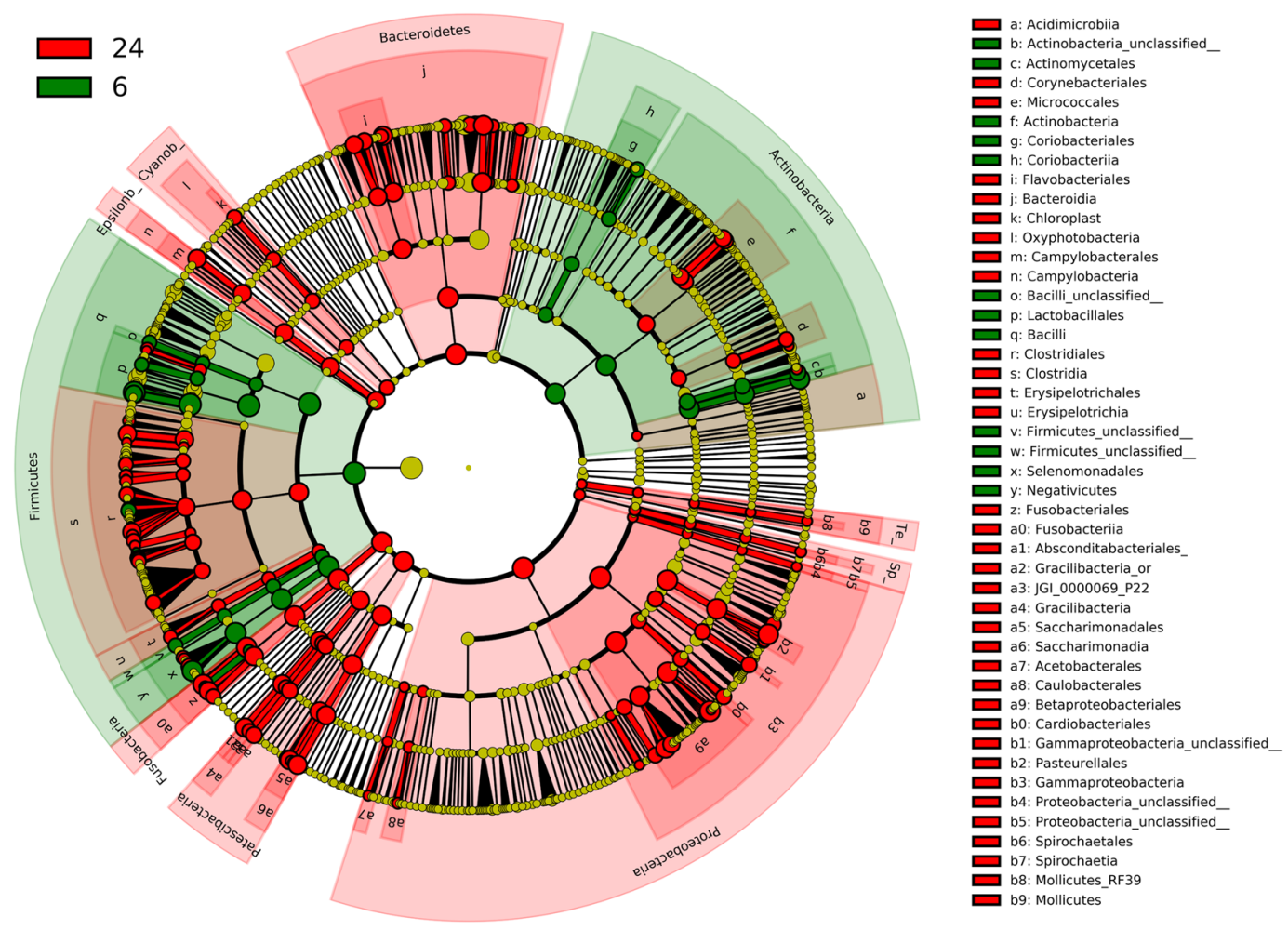

Figure 4. Cladogram showing differentially expressed bacteria at 6 and 24 months, respectively. Green colour indicates higher abundance at 6 months and red colour higher abundance at 24 months. Inner circle represents phyla and outer circles lower taxonomic levels.

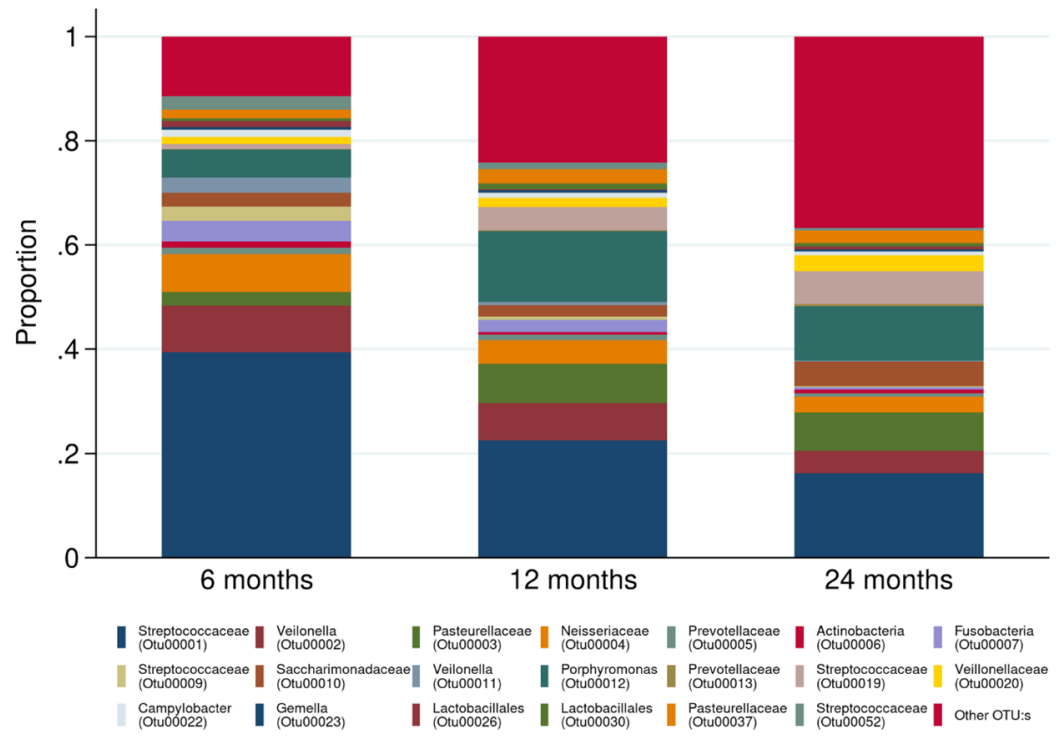

Figure 5. Stacked bar charts of the relative distribution of the 20 most abundant operational taxonomic units (OTUs) at 6, 12, and 24 months, respectively. Other OTUs constituted $11.5 \%, 24.3 \%$, and $36.8 \%$ of the total amount of reads (at 6,12 and 24 months respectively).

age (Fig. 6). Nevertheless, at 24 months, the microbiota of each child did not resemble their own mother's microbiotaduring pregnancy more than any of the other samples of the maternal cohort $(p=0.17)$.

Birth characteristics and breastfeeding. Gestational age and exclusive breastfeeding wereboth associated with overall phyla distribution $(\mathrm{p}<0.01$, Table S2). However, no specific phyla were identified in post-hoc analyses that could subsequently be confirmed in semi-parametric sensitivity analyses (Table S3). Gestational age and exclusive breastfeeding were also associated with beta diversity $(\mathrm{p}=0.003$ and $\mathrm{p}=0.02$, respectively). 


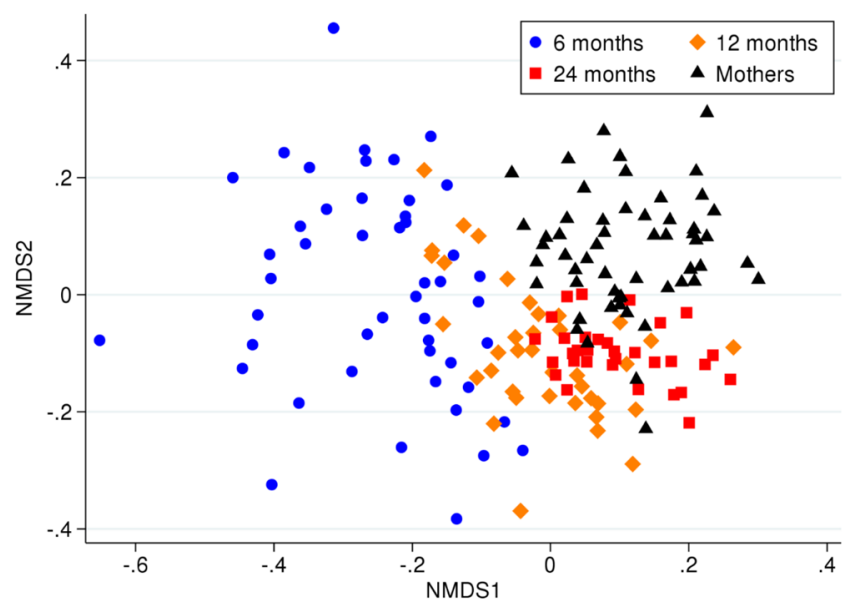

Figure 6. NMDS illustrating the children's beta diversity across the three time points, as well as maternal beta diversity during pregnancy.

Being born at an older gestational age was associated with an increase in the relative abundance of an OTU within the Saccharimonadacea genus (OTU00010, $\mathrm{p}<0.001$ ) but with a decrease of an OTU belonging to the Porphyromonas genus (OTU00012, $\mathrm{p}=0.01$, Fig. 7). Birth weight exhibited $\mathrm{u}$-shaped associations with two OTUs within the Veillonella genus (OTU0002, $\mathrm{p}=0.003$ and OTU00011, $\mathrm{p}<0.001$ ). We also observed inverted $\mathrm{u}$-shaped associations between birth weight and two OTUs belonging to the Lactobacillales order (OTU00026, $\mathrm{p}=0.001$ and OTU00030, $\mathrm{p}=0.02$, Fig. 7). We furthermore observed an interaction between birth weight and age for the presence of one of the Veillonella OTUs (OTU00011), where a birth weight below the $50^{\text {th }}$ percentile entailed a lower abundance noted foremost at 6 months of age $(\mathrm{p}<0.001$ : Fig. S3). We found no associations between parity or mode of delivery and oral microbiota of the child.

Morning salivary cortisol, antibiotic treatment, and pet exposure. Overall, we observed similar median morning salivary cortisol levels across all time points. Both morning salivary cortisol level and antibiotic treatment within the last 90 days of the saliva microbiota assessments were associated with proportion of phyla $(\mathrm{p}=0.003$ and $\mathrm{p}=0.008$, respectively), but no specific phyla were identified in post-hoc analyses that could also be confirmed in semi-parametric sensitivity analyses.

Morning salivary cortisol was inversely associated the relative abundance of the most common OTU mapped to the Streptococcaceae family (OTU0001, $\mathrm{p}=0.02$ ). This association was mainly driven be the group of children with cortisol values over $25 \mathrm{nmol} /$ liter (Fig. 7).

Antibiotics treatment within the last 90 days was positively associated with two OTUs belonging to the Pasteurellaceae and Neisseriaceae families (OTU00003, RPR 1.84; $\mathrm{p}=0.005$ and OTU00004, RPR 1.84; $\mathrm{p}=0.01$, respectively) but negatively associated with an OTU within the Prevotellaceae family (OTU00013, RPR 0.07; $\mathrm{p}<0.001)$. For the Prevotellaceae OTU we further noted an interaction between antibiotics prescribed within 90 days and age (OTU00013, $\mathrm{p}=0.03$ : Fig. S3), with the lower abundance noted mainly at six months. We observed no association between ever-use of antibiotics and oral microbiota outcomes. Lastly, presence of a furry pet in the household was associated with a lower relative abundance of an OTU belonging to the Pasteurellaceae family (OTU00003, RPR 0.46; $\mathrm{p}=0.01$ ).

\section{Discussion}

In this longitudinal population-based cohort study of 59 Swedish children, our main findings were twofold. First, we observed a radical shift in the salivary microbiota composition from 6 months to 24 months of age, including a nearly threefold increase in alpha diversity as well as profound changes on all investigated taxonomic levels. Secondly, we found a novel link between salivary cortisol and oral microbiota composition, encompassing both phyla distribution and lower relative abundance of the most common oral OTU belonging to the Streptococcaceae family. In addition, we also found evidence of associations between birth characteristics as well as other early life exposures with oral microbiota composition. We specifically noted a transient association between antibiotic treatment and the oral microbiome.

We observed a steady increase of oral microbiota alpha diversity associated with increasing age, indicating that both richness and species distribution evenness in individual saliva samples increased over time. This finding aligns with a recent Swedish longitudinal study of children with a family history of allergic disease that reported a positive association between age and oral microbiome alpha diversity ${ }^{17}$. At 6 months, we observed a vast dominance of Firmicutes, largely attributed to members of the Streptococci family. This is concordant with previous studies reporting a Firmicutes dominance at $4-8$ weeks ${ }^{14}, 3$ months ${ }^{15}$, and 6 months of age ${ }^{16}$. The subsequent decrease of Firmicutes, accompanied by an increase in other phyla including Proteobacteria, also corresponds with the findings of two earlier studies which utilized pyrosequencing to examine saliva samples from 3-year old children $^{3,15}$. We further found that the beta diversity of the children decreased with age and also that children's 

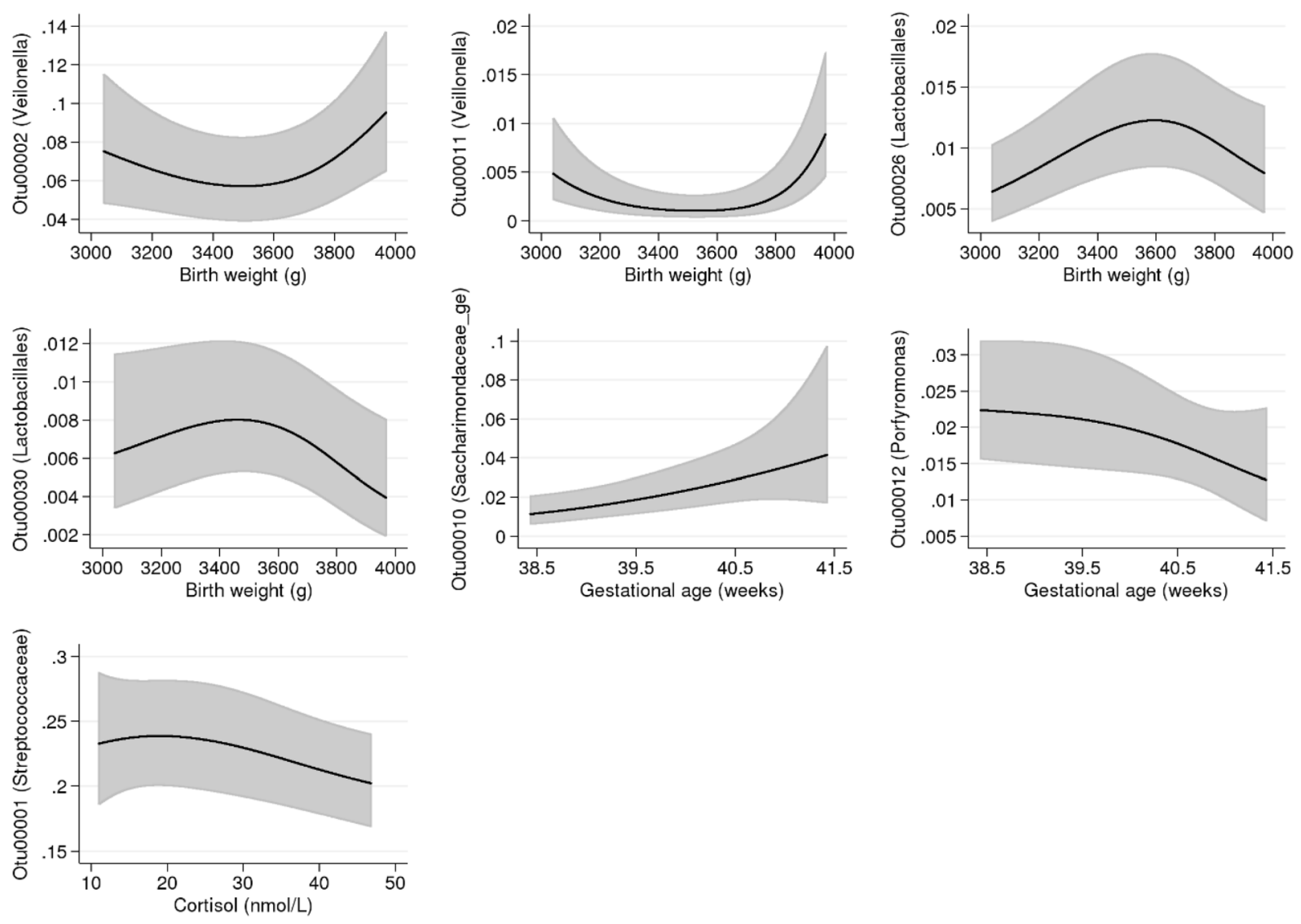

Figure 7. Relative abundance of specific operational taxonomic units (OTUs) associated with the continuous variables birth weight, gestational age and morning salivary cortisol. Lines indicate predicted mean values and $95 \%$ confidence intervals, adjusted for age and potential confounders (see Table 1). All continuous adjustment variables are fixed at their mean value and all categorical adjustment variables are fixed at their most common category. The $\mathrm{x}$-axis ranges from the 10th to the 90 th percentile of the variable. The $y$-axis shows the relative abundance in proportion.

samples became more similar to that of the maternal cohort over time, suggesting both that the initially larger inter-individual differences were attenuated over time, and that the children had reached a more adult-like composition at 24 months. This oral microbiota maturation process is likely influenced by several factors, including the emergence of deciduous teeth which creates new micro-environments and niches for oral bacterial colonization and microfilm adherence ${ }^{43}$ and changes in diet including the introduction of solid foods and an increase in variation of $\operatorname{diet}^{44}$. Additionally, an increased horizontal transmission may also occur as the child is increasingly exposed to a greater multitude of new people and environments, including day-care, during the first years of life. In Sweden, children start day-care at 12 months or later. On the OTU-level, we specifically noted increased relative abundances of OTUs within the Porphyromonas and the Fusobacterium genera with increasing age. The Porphyromonas genus include Porphyromonas gingivalis, implicated in periodontal disease but also previously detected in oral cavities of healthy children ${ }^{45}$, whilst the Fusobacterium genus include the bacteria Fusobacterium nucleatum, a species widely present in the oral cavity but also considered to be an opportunistic oral pathogen ${ }^{46}$. However, as our analysis pipe-line did not allow for species-level taxonomic resolution, we cannot discern the presence of these, or other potentially pathogenic, bacterial species within our samples.

We noted temporal similarities between our findings and previous reports on the development of the gut microbiota composition across the first years of life. As in the oral cavity, alpha diversity increases dramatically in the gut before the age of 24 months, with an adult-like composition reached at 2-3 years ${ }^{47-49}$. A longitudinal study of European and US children reported three distinct phases of gut microbiome progression, with an initial rapid development phase (3-14 months) characterized by major changes in the abundance of all five main gut phyla, a transitional phase (15-30 months) with distinct changes found only for two of the five main phyla, and a stable phase ( $>30$ months) where little phyla redistribution occurred ${ }^{50}$. In correspondence, we observed the steepest changes in our two most abundant oral phyla Firmicutes and Proteobacteria in the first year of life, whilst less abrupt changes transpired in the children's second year.

We could not detect any association between mode of delivery and oral microbiota. Caesarean sections has previously been noted as an important determinant for oral microbiota composition in newborns ${ }^{12}$. Our findings however align with the results from a longitudinal $16 \mathrm{~S}$ rRNA gene sequencing study of 81 infants, which did observe an association immediately after birth, but which could not detect any association at follow-up sampling 
at 6 weeks of age ${ }^{11}$. In contrast, other studies have reported that caesarean section may be associated with a lower number of oral bacterial taxa at 3 months ${ }^{51}$, and also with beta diversity at 6 months ${ }^{17}$, although the latter association was largely attenuated after adjustment for age, breastfeeding and later caries status. The discrepant results on the potential association between mode of delivery and oral microbiota likely reflect both that the influence of mode of delivery diminish over time and are therefore not detectable in our cohort where the first saliva sampling was performed at 6 months of age, as well as methodological dissimilarities between different study designs including adjustments for potential confounders.

We noted an association between duration of exclusive breastfeeding and a difference in overall phyla distribution, but could not identify any specific phyla or OTU associated with exclusive breastfeeding. Breastfeeding has previously been associated with abundance of Actinobacteria and Proteobacteria at 4-8 weeks ${ }^{14}$, and prevalence of Lactobacillus at 3 months ${ }^{52}$. An important limitation of our material is however that the upper pre-determined age-category for exclusive breastfeeding was $5+$ months in the Born into Life questionnaire. Even children who were exclusively breast-fed for $5+$ months may therefore have been introduced to solid foods and/or formula at the time of the first saliva sampling (at 6 months). Overall, the children in our cohort were also older and more likely to have deciduous teeth. The potential influence of breastfeeding on the oral microbiota has thereby likely been attenuated in our study. In addition, there are again also methodological differences between the previous studies and ours, as we have adjusted the statistical model for exclusive breastfeeding for several potential confounders identified through our DAG (Table 1), covariates that have not been included in the previous studies.

We observed that gestational age was associated with overall phyla distribution, and both gestational age and birth weight were associated with specific OTUs, including an inverted u-shaped associations between birth weight and two OTUs belonging to the Lactobacillales order. An American cohort study of 40 neonates recently reported that children born at a young gestational age with a low birth weight exhibited a greater abundance of the two genera Stenotrophomonas and Enterobacter, both within the Gammaproteobacteria class, as well as Lactococcus, a bacterial genus within the Lactobacillales order, as compared with term-born infants ${ }^{9}$. Although both studies note an influence of birth weight and gestational age on bacterial taxa within the Lactobacillales order, direct comparisons between the two studies may not be feasible as the American study only included very preterm infants sampled at the neonatal intensive care unit. In our study, being born at an older gestational age was also associated with an increase in the relative abundance of an OTU within the Saccharimonadacea genus (OTU00010), a genus belonging to the Patescibacteria phyla, a newly defined major microbial group within the updated topology of the bacterial domain ${ }^{53}$. No cultured isolates are available of the Saccharimonadacea genus as it has only been encountered through sequencing, and its function remains unclear. A recent study reported a higher abundance of Saccharimonadacea in non-caries afflicted adults than in adult with caries ${ }^{54}$. Further studies will however be needed to interpret our finding in association with gestational age.

To the best of our knowledge, no previous study has explored the association between naturally occurring variation in salivary cortisol and oral microbiota community composition. We observed that increased level of cortisol was associated with changes in phyla distribution, and a lower relative abundance of a common OTU belonging to the Streptococcaceae family. The association with the Streptococcaceae OTU was mainly seen in children with cortisol concentrations $>25 \mathrm{nmol} / \mathrm{L}$. Healthy Norwegian children have been reported to have a mean salivary cortisol of $28.7 \mathrm{nmol} / \mathrm{L}$ at 6 months, and $34.9 \mathrm{nmol} / \mathrm{L}$ at 24 months ${ }^{55}$. Cortisol has previously been found to alter in vitro growth of periodontal-related bacterial strains ${ }^{56}$. Our findings support that salivary cortisol is also associated with oral microbiota community composition in vivo.

Although we both applied a false discovery rate threshold, and filtered out results based on the more robust ordinal logistic regression in our analyses, we cannot exclude the possibility that random chance alone was responsible for our noted associations between salivary cortisol and oral microbiota. Further studies are needed to confirm our finding. We also adjusted all analyses of the associations between salivary cortisol and microbiota for the potential confounders available in the Born into Life study including maternal education level and birth characteristics, but we did not have access to information on other possible confounders including sleep patterns and dietary timing, as well as home and childcare environmental circumstances. Residual confounding may therefore still be present. Further, even though it may be of interest to investigate the association between cortisol circadian rhythm and saliva microbiota, we did not include measurements of evening salivary cortisol in our study in order minimize the risk of a matrix effect. Lastly, our study is cross-sectional in nature, and though we primarily hypothesize that cortisol may influence microbiota composition and development we cannot attempt to infer any causal direction. A reverse causation where oral microbiota may influence neuroendocrine activity and cortisol secretion remains possible.

If our associations are truly causal, we hypothesize that there could be different, potentially non-exclusive, pathways linking cortisol with oral bacterial taxa. The concept of a microbiome-gut-brain axis, with bidirectional communication between neuroendocrine activation including the hypothalamic-pituitary-adrenal axis and gut microbiota composition and function, is currently discussed within the gut microbiota research field ${ }^{57-60}$. Suggested mechanisms for the microbiome-gut-brain axis include a microbiota-driven pro-inflammatory activation of the hypothalamic-pituitary-adrenal axis, and an influence of cortisol on gut microbiota composition and gastrointestinal permeability. In parallel to this, we speculate that our results could indicate that the oral microbiota composition and development in early childhood may be influenced by systemic neuroendocrine activation. Another potential mechanism is that salivary cortisol could influence bacterial growth and biofilm formation in the oral cavity through local immunomodulation. Although longitudinal data is lacking, smaller cross-sectional studies have reported that salivary cortisol may be associated with the development of dental caries in childhood, and also with a larger number of cariogenic bacteria ${ }^{19-21}$, the latter including Streptococcus mutans and Lactobacillus species, bacteria involved in tooth decay and caries progression, respectively. Unfortunately, due to limitations in our analysis pipeline, we cannot discern if the cortisol-associated Streptococcaceae OTU in the present study represents Streptococcus mutans. 
We further found that exposure to antibiotics within 90 days of saliva sampling was associated with proportion of phyla, as well as positively associated with OTUs belonging to the Pasteurellaceae and Neisseriaceae families and negatively associated with a OTU within the Prevotellaceae family. The most common indication for antibiotic treatment in young children in Sweden is a respiratory tract infection ${ }^{61,62}$ including acute otitis media, with national guidelines recommending treatment with a $\beta$-lactam antibiotics for 5-10 days. Although we do not have access to treatment duration for the children in the Born into Life cohort, phenoxylmethylpenicillin and amoxicillin represented the most commonly prescribed antibiotics mirroring the national guidelines. In interpreting our findings, it is also noteworthy that antibiotics are most often prescribed as oral suspensions to young children, with potential local effects in the oral cavity.

The Pasteurellaceae and Neisseriaceae families contain known pathogens including Pasteurella multocida and Neisseria meningitidis. However, although we cannot determine if these species are included in the OTUs we have detected, these pathogens are sensitive to phenoxylmethylpenicillin and amoxicillin, and should not have survived the antibiotics treatment. A Swiss cohort study using pyrosequencing found that amoxicillin prescribed for otitis media in children was associated with a reduced species richness and diversity in the oral cavity, and with a major shift in relative abundance of several taxa at the end of the antibiotic treatment ${ }^{63}$. However, three weeks after the amoxicillin treatment ended, the saliva microbiota had largely recovered to the composition present before treatment commenced ${ }^{63}$. Corresponding results were reported from a randomized controlled trial on healthy adults ${ }^{64}$, which found that antibiotic treatment had extensive and long-term effects on gut microbiota, whereas alternations of oral microbiota after exposure to antibiotics were of lower magnitude and transient. A swift recovery of oral microbiota after antibiotic treatment cessation may explain why we could not distinguish any association between ever-use of antibiotics and oral microbiota in our cohort.

Lastly, we also noted that presence of a furry pet in the household was associated with a lower relative abundance of an OTU within the Pasteurellaceae family, a bacterial family containing strains that are potential zoonotic agents and are usually present in cats and dogs. The direction of the association was surprising and further studies are needed to confirm the finding.

Strengths and limitations. A strength of this study is the longitudinal design, which allowed for repeated assessments of oral microbiota and salivary cortisol, as well as for time-updated information on early childhood exposures. Linkages to national health registers furthermore enabled access to objectively recorded maternal and birth characteristics, and also to detailed information on antibiotic prescription without any risk of recall bias. The identification of potential confounders for each exposure before the analysis phase of the study further reduced the overall risk of bias.

In addition to the limitations discussion in association with our findings on salivary cortisol, a few more potential weaknesses of our study however also deserve mentioning. Firstly, our platform for 16s rRNA amplicon sequencing did not allow for a species-level taxonomic resolution in OTUs. Interpreting potential pathogenic effects of our associations is therefore challenging. Secondly, the time-points for questionnaires and biomarker sampling were pre-determined for the Born into Life cohort, and unfortunately no saliva sampling was performed in the neonatal period. Further, our sample size of 59 mother-child pairs had limited power to detect associations for rarer exposures such as caesarean section, antibiotic treatment and furry pet in our cohort, and also to identify changes in specific phyla contributing to an overall change in phyla distribution. Also, fewer children participated in the examinations at 12 and 24 months than at 6 months which may have further reduced the possibility to detect associations present only at older age. Finally, the maternal cohort represents a well-educated population who had originally volunteered to participate in LifeGene and then also accepted to partake in Born into Life whilst pregnant, and the child cohort consists of mainly term-born healthy children. This limited our possibility to observe for example associations between unfavourable maternal health behaviours and the child's oral microbiota, and may also restrict the generalizability of our findings. However, expanding the knowledge base of the oral microbiota development trajectory in a healthy context may enable future identification of adverse oral microbiota markers in children born in less advantageous environments.

In conclusion, the findings of this longitudinal study include a novel association between salivary cortisol and oral microbiota and a transient effect of antibiotics on the oral microbiome in early childhood. It also highlights the radical shifts in the oral microbiota composition from 6 months to 24 months of age. Future studies utilizing metagenome shotgun sequencing may enable an increased accuracy and a more detailed information on bacterial function.

\section{Data availability}

Restrictions apply to the availability of individual level health data, which were used under license and ethical approval for the current study, and are not publicly available. Individual level data are however available from the authors upon reasonable request and with permission of the Swedish Ethical Review Authority. Bacterial sequencing data has been submitted to the Sequence Read Archive (SRA) open repository under reference number PRJNA575550.

Received: 4 July 2019; Accepted: 14 November 2019;

Published online: 13 December 2019

\section{References}

1. Xu, X. et al. Oral cavity contains distinct niches with dynamic microbial communities. Environ Microbiol 17, 699-710, https://doi. org/10.1111/1462-2920.12502 (2015).

2. Takahashi, N. \& Nyvad, B. The role of bacteria in the caries process: ecological perspectives. J Dent Res 90, 294-303, https://doi. org/10.1177/0022034510379602 (2011).

3. Crielaard, W. et al. Exploring the oral microbiota of children at various developmental stages of their dentition in the relation to their oral health. BMC Med Genomics 4, 22, https://doi.org/10.1186/1755-8794-4-22 (2011). 
4. Slocum, C., Kramer, C. \& Genco, C. A. Immune dysregulation mediated by the oral microbiome: potential link to chronic inflammation and atherosclerosis. J Intern Med 280, 114-128, https://doi.org/10.1111/joim.12476 (2016).

5. Dzidic, M. et al. Oral microbiota maturation during the first 7 years of life in relation to allergy development. Allergy 73, 2000-2011, https://doi.org/10.1111/all.13449 (2018).

6. Craig, S. J. C. et al. Child Weight Gain Trajectories Linked To Oral Microbiota Composition. Sci Rep 8, 14030, https://doi. org/10.1038/s41598-018-31866-9 (2018).

7. Qiao, Y. et al. Alterations of oral microbiota distinguish children with autism spectrum disorders from healthy controls. Sci Rep 8, 1597, https://doi.org/10.1038/s41598-018-19982-y (2018).

8. Gomez-Arango, L. F. et al. Antibiotic treatment at delivery shapes the initial oral microbiome in neonates. Sci Rep 7, 43481, https:// doi.org/10.1038/srep43481 (2017).

9. Younge, N. E., Araujo-Perez, F., Brandon, D. \& Seed, P. C. Early-life skin microbiota in hospitalized preterm and full-term infants. Microbiome 6, 98, https://doi.org/10.1186/s40168-018-0486-4 (2018).

10. Sohn, K., Kalanetra, K. M., Mills, D. A. \& Underwood, M. A. Buccal administration of human colostrum: impact on the oral microbiota of premature infants. J Perinatol 36, 106-111, https://doi.org/10.1038/jp.2015.157 (2016).

11. Chu, D. M. et al. Maturation of the infant microbiome community structure and function across multiple body sites and in relation to mode of delivery. Nat Med 23, 314-326, https://doi.org/10.1038/nm.4272 (2017).

12. Dominguez-Bello, M. G. et al. Delivery mode shapes the acquisition and structure of the initial microbiota across multiple body habitats in newborns. Proc Natl Acad Sci USA 107, 11971-11975, https://doi.org/10.1073/pnas.1002601107 (2010).

13. Li, H. et al. The impacts of delivery mode on infant's oral microflora. Sci Rep 8, 11938, https://doi.org/10.1038/s41598-018-30397-7 (2018).

14. Al-Shehri, S. S. et al. Deep sequencing of the $16 \mathrm{~S}$ ribosomal RNA of the neonatal oral microbiome: a comparison of breast-fed and formula-fed infants. Sci Rep 6, 38309, https://doi.org/10.1038/srep38309 (2016).

15. Lif Holgerson, P., Ohman, C., Ronnlund, A. \& Johansson, I. Maturation of Oral Microbiota in Children with or without Dental Caries. PLoS One 10, e0128534, https://doi.org/10.1371/journal.pone.0128534 (2015).

16. Drell, T. et al. The Influence of Different Maternal Microbial Communities on the Development of Infant Gut and Oral Microbiota. Sci Rep 7, 9940, https://doi.org/10.1038/s41598-017-09278-y (2017).

17. Dzidic, M. et al. Oral microbiome development during childhood: an ecological succession influenced by postnatal factors and associated with tooth decay. ISME J 12, 2292-2306, https://doi.org/10.1038/s41396-018-0204-z (2018).

18. McEwen, B. S. Protective and damaging effects of stress mediators. N Engl J Med 338, 171-179, https://doi.org/10.1056/ NEJM199801153380307 (1998)

19. Boyce, W. T. et al. Social inequalities in childhood dental caries: the convergent roles of stress, bacteria and disadvantage. Soc Sci Med 71, 1644-1652, https://doi.org/10.1016/j.socscimed.2010.07.045 (2010).

20. Barbosa, T. S., Castelo, P. M., Leme, M. S. \& Gaviao, M. B. Associations between oral health-related quality of life and emotional statuses in children and preadolescents. Oral Dis 18, 639-647, https://doi.org/10.1111/j.1601-0825.2012.01914.x (2012).

21. Pani, S. C., Abuthuraya, D., Alshammery, H. M., Alshammery, D. \& Alshehri, H. Salivary cortisol as a biomarker to explore the role of maternal stress in early childhood caries. Int J Dent 2013, 565102, https://doi.org/10.1155/2013/565102 (2013).

22. Smew, A. I. et al. Limited association between markers of stress during pregnancy and fetal growth in 'Born into Life', a new prospective birth cohort. Acta Paediatr 107, 1003-1010, https://doi.org/10.1111/apa.14246 (2018).

23. Almqvist, C. et al. LifeGene-a large prospective population-based study of global relevance. Eur J Epidemiol 26, 67-77, https://doi. org/10.1007/s10654-010-9521-x (2011).

24. Chiu, M. L. et al. Matrix Effects-A Challenge toward Automation of Molecular Analysis. JALA: Journal of the Association for Laboratory Automation 15, 233-242, https://doi.org/10.1016/j.jala.2010.02.001 (2010).

25. Schloss, P. D. et al. Introducing mothur: open-source, platform-independent, community-supported software for describing and comparing microbial communities. Appl Environ Microbiol 75, 7537-7541, https://doi.org/10.1128/AEM.01541-09 (2009).

26. Kozich, J. J., Westcott, S. L., Baxter, N. T., Highlander, S. K. \& Schloss, P. D. Development of a dual-index sequencing strategy and curation pipeline for analyzing amplicon sequence data on the MiSeq Illumina sequencing platform. Appl Environ Microbiol 79, 5112-5120, https://doi.org/10.1128/AEM.01043-13 (2013).

27. Rognes, T., Flouri, T., Nichols, B., Quince, C. \& Mahe, F. VSEARCH: a versatile open source tool for metagenomics. PeerJ 4, e2584, https://doi.org/10.7717/peerj.2584 (2016).

28. Shrier, I. \& Platt, R. W. Reducing bias through directed acyclic graphs. BMC Med Res Methodol 8, 70, https://doi.org/10.1186/14712288-8-70 (2008)

29. Greenland, S., Pearl, J. \& Robins, J. M. Causal diagrams for epidemiologic research. Epidemiology 10, 37-48 (1999).

30. Benjamini, Y. \& Hochberg, Y. Controlling the False Discovery Rate: A Practical and Powerful Approach to Multiple Testing. Journal of the Royal Statistical Society: Series B (Methodological) 57, 289-300, https://doi.org/10.1111/j.2517-6161.1995.tb02031.x (1995).

31. Harrell, F. E. In Regression modeling strategies: with applications to linear models, logistic regression, and survival analysis xxii, $568 \mathrm{p}$. (Springer, 2001).

32. Simpson, E. Measurement of Diversity. Nature (1949).

33. Lanzén, A. et al. Multi-targeted metagenetic analysis of the influence of climate and environmental parameters on soil microbial communities along an elevational gradient. Scientific Reports 6, 28257, https://doi.org/10.1038/srep28257 (2016)

34. Ochoa-Franco, Ad. P. et al. Beta-Diversity Modeling and Mapping with LiDAR and Multispectral Sensors in a Semi-Evergreen Tropical Forest. Forests 10, 419 (2019).

35. Sandau, N. et al. Including community composition in biodiversity-productivity models. Methods in Ecology and Evolution $\mathbf{5}$, 815-823, https://doi.org/10.1111/2041-210x.12215 (2014).

36. Legendre, P., Legendre, L., Legendre, L. \& Legendre, L. Numerical ecology. 2nd English edn, (Elsevier, 1998).

37. Papke, L. E. \& Wooldridge, J. M. Econometric methods for fractional response variables with an application to 401(k) plan participation rates. 11, 619-632, 10.1002/(SICI) 1099-1255(199611)11:6<619::AID-JAE418>3.0.CO;2-1 (1996).

38. Buis, M. L. FMLOGIT: Stata module fitting a fractional multinomial logit model by quasi maximum likelihood. Statistical Software Components S456976. Boston College Department of Economics (2008)

39. Anderson, M. J. A new method for non-parametric multivariate analysis of variance. Austral Ecology 26, 32-46, https://doi.org/10. 1111/j.1442-9993.2001.01070.pp.x (2001).

40. Hervé, M. RVAideMemoire: Testing and Plotting Procedures for Biostatistics. R package version 0.9-73 (2019).

41. Segata, N. et al. Metagenomic biomarker discovery and explanation. Genome Biol 12, R60, https://doi.org/10.1186/gb-2011-12-6-r60 (2011).

42. Afgan, E. et al. The Galaxy platform for accessible, reproducible and collaborative biomedical analyses: 2018 update. Nucleic Acids Res 46, W537-W544, https://doi.org/10.1093/nar/gky379 (2018).

43. Kononen, E. Development of oral bacterial flora in young children. Ann Med 32, 107-112 (2000).

44. Klingberg, S., Ludvigsson, J. \& Brekke, H. K. Introduction of complementary foods in Sweden and impact of maternal education on feeding practices. Public Health Nutr 20, 1054-1062, https://doi.org/10.1017/S1368980016003104 (2017).

45. Kulekci, G., Leblebicioglu, B., Keskin, F., Ciftci, S. \& Badur, S. Salivary detection of periodontopathic bacteria in periodontally healthy children. Anaerobe 14, 49-54, https://doi.org/10.1016/j.anaerobe.2007.08.001 (2008). 
46. Brennan, C. A. \& Garrett, W. S. Fusobacterium nucleatum - symbiont, opportunist and oncobacterium. Nat Rev Microbiol 17, 156-166, https://doi.org/10.1038/s41579-018-0129-6 (2019).

47. Yatsunenko, T. et al. Human gut microbiome viewed across age and geography. Nature 486, 222-227, https://doi.org/10.1038/ nature11053 (2012).

48. Hesla, H. M. et al. Impact of lifestyle on the gut microbiota of healthy infants and their mothers-the ALADDIN birth cohort. FEMS Microbiol Ecol 90, 791-801, https://doi.org/10.1111/1574-6941.12434 (2014).

49. Bokulich, N. A. et al. Antibiotics, birth mode, and diet shape microbiome maturation during early life. Sci Transl Med 8, 343ra382, https://doi.org/10.1126/scitranslmed.aad7121 (2016)

50. Stewart, C. J. et al. Temporal development of the gut microbiome in early childhood from the TEDDY study. Nature 562, 583-588, https://doi.org/10.1038/s41586-018-0617-x (2018).

51. Lif Holgerson, P., Harnevik, L., Hernell, O., Tanner, A. C. \& Johansson, I. Mode of birth delivery affects oral microbiota in infants. J Dent Res 90, 1183-1188, https://doi.org/10.1177/0022034511418973 (2011).

52. Holgerson, P. L. et al. Oral microbial profile discriminates breast-fed from formula-fed infants. J Pediatr Gastroenterol Nutr 56, 127-136, https://doi.org/10.1097/MPG.0b013e31826f2bc6 (2013).

53. Castelle, C. J. \& Banfield, J. F. Major New Microbial Groups Expand Diversity and Alter our Understanding of the Tree of Life. Cell 172, 1181-1197, https://doi.org/10.1016/j.cell.2018.02.016 (2018).

54. Schoilew, K. et al. Bacterial biofilm composition in healthy subjects with and without caries experience. J Oral Microbiol 11, 1633194, https://doi.org/10.1080/20002297.2019.1633194 (2019).

55. Rolfsjord, L. B. et al. Morning Salivary Cortisol in Young Children: Reference Values and the Effects of Age, Sex, and Acute Bronchiolitis. J Pediatr 184, 193-198 e193, https://doi.org/10.1016/j.jpeds.2017.01.064 (2017).

56. Jentsch, H. F., Marz, D. \& Kruger, M. The effects of stress hormones on growth of selected periodontitis related bacteria. Anaerobe 24, 49-54, https://doi.org/10.1016/j.anaerobe.2013.09.001 (2013).

57. Kelly, J. R. et al. Breaking down the barriers: the gut microbiome, intestinal permeability and stress-related psychiatric disorders. Front Cell Neurosci 9, 392, https://doi.org/10.3389/fncel.2015.00392 (2015).

58. Zhao, L. et al. Bidirectional gut-brain-microbiota axis as a potential link between inflammatory bowel disease and ischemic stroke. J Neuroinflammation 15, 339, https://doi.org/10.1186/s12974-018-1382-3 (2018).

59. Farzi, A., Frohlich, E. E. \& Holzer, P. Gut Microbiota and the Neuroendocrine System. Neurotherapeutics 15, 5-22, https://doi. org/10.1007/s13311-017-0600-5 (2018).

60. Huang, T. T. et al. Current Understanding of Gut Microbiota in Mood Disorders: An Update of Human Studies. Front Genet 10, 98, https://doi.org/10.3389/fgene.2019.00098 (2019).

61. Hellman, J., Grape, M. \& Ternhag, A. Antibiotic consumption among a Swedish cohort of children born in 2006. Acta Paediatr 104, 1035-1038, https://doi.org/10.1111/apa.13097 (2015).

62. Andre, M. et al. The management of infections in children in general practice in Sweden: a repeated 1-week diagnosis-prescribing study in 5 counties in 2000 and 2002. Scand J Infect Dis 37, 863-869, https://doi.org/10.1080/00365540500335207 (2005).

63. Lazarevic, V. et al. Effects of amoxicillin treatment on the salivary microbiota in children with acute otitis media. Clin Microbiol Infect 19, E335-342, https://doi.org/10.1111/1469-0691.12213 (2013).

64. Zaura, E. et al. Same Exposure but Two Radically Different Responses to Antibiotics: Resilience of the Salivary Microbiome versus Long-Term Microbial Shifts in Feces. MBio 6, e01693-01615, https://doi.org/10.1128/mBio.01693-15 (2015).

\section{Acknowledgements}

The authors wish to thank Dr Susanne Hetty, Department of Medical Sciences, Uppsala University, for her excellent advice on saliva sample preparation and DNA extraction, Dr Janet L. Cunningham, Department of Neuroscience, Uppsala University, for generously sharing her expertise on microbial endocrinology, and Professor Hilpi Rautelin, Department of Medical Sciences, Uppsala University, for her highly valuable input on potential oral consequences of antibiotic treatment. The computations were performed on resources provided by SNIC through Uppsala Multidisciplinary Center for Advanced Computational Science (UPPMAX) under Project SNIC sens2018616. The authors further wish to acknowledge the support of the National Genomics Infrastructure (NGI)/Uppsala Genome Center. Work performed at NGI/Uppsala Genome Center has been funded by RFI/VR and Science for Life Laboratory, Sweden, and to thank the Biobank at Karolinska Institutet for professional and qualitative biobank services. Illumina sequencing was performed by the SNP\&SEQ Technology Platform in Uppsala. The facility is part of NGI Sweden and Science for Life Laboratory. The SNP\&SEQ Platform is also supported by the Swedish Research Council and the Knut and Alice Wallenberg Foundation. Financial support was provided from the Swedish Research Council through grants to Professor Tove Fall (no 2015-03477), Professor Göran Pershagen (no 2015-02434_3), and Professor Catarina Almqvist (no 2018-02640) as well as from the Swedish Initiative for Research on Microdata in the Social And Medical Sciences (SIMSAM) framework grant (no 340-2013-5867). Grants have also been provided by the Stockholm County Council (ALF-projects), the Strategic Research Program in Epidemiology (SFO-epi) at Karolinska Institutet, the Swedish Heart-Lung Foundation, the Swedish Asthma and Allergy Association's Research Foundation, the Swedish Research Council for Health, Working Life and Welfare (FORTE, no 2015-00289), and the Department of Clinical Sciences at Danderyd Hospital. The project was further supported by the strategic research area (SFO) EXODIAB and the Uppsala Antibiotic Center. Open access funding provided by Uppsala University.

\section{Author contributions}

B.K., S.P., U.H., S.V., A.H., C.A., E.A., G.P., J.D., S.B. and T.F. devised the hypothesis and designed the study. E.A., C.A. and A.H. acquired the data, U.H. performed the statistical analyses, and B.K. conducted the literature review and wrote the first draft. T.F. is the study supervisor. B.K., S.P., U.H., S.V., A.H., C.A., E.A., G.P., J.D., S.B. and T.F. participated in critical revision of the manuscript, and have had full access to all of the data in the study.

\section{Competing interests}

The authors declare no competing interests.

\section{Additional information}

Supplementary information is available for this paper at https://doi.org/10.1038/s41598-019-54702-0.

Correspondence and requests for materials should be addressed to B.K. 
Reprints and permissions information is available at www.nature.com/reprints.

Publisher's note Springer Nature remains neutral with regard to jurisdictional claims in published maps and institutional affiliations.

(c) (i) Open Access This article is licensed under a Creative Commons Attribution 4.0 International License, which permits use, sharing, adaptation, distribution and reproduction in any medium or format, as long as you give appropriate credit to the original author(s) and the source, provide a link to the Creative Commons license, and indicate if changes were made. The images or other third party material in this article are included in the article's Creative Commons license, unless indicated otherwise in a credit line to the material. If material is not included in the article's Creative Commons license and your intended use is not permitted by statutory regulation or exceeds the permitted use, you will need to obtain permission directly from the copyright holder. To view a copy of this license, visit http://creativecommons.org/licenses/by/4.0/.

(C) The Author(s) 2019 